\title{
Single nucleotide polymorphisms in NOS2A and NOS3 genes are not associated with treatment response of non-small cell lung cancer patients following the definitive radiochemotherapy
}

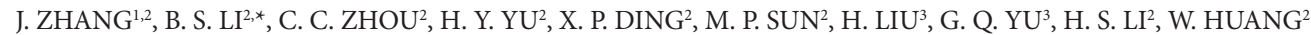

${ }^{1}$ Department of Radiation Oncology, Cancer Hospital, Tianjin Medical University, Huan-Hu-Xi Road, Tianjin, 30060, P.R.China; ${ }^{2}$ Shandong Cancer Hospital, Shandong Academy of Medical Sciences, 440, Jiyan Road, Jinan, 250117, P.R.China; ${ }^{3}$ Shandong Provinical Institute of Dermatology and Venereology, Jingshi Road, Jinan, 250022, P.R.China

*Correspondence: baoshli@yahoo.com

Received April 2, 2012 / Accepted May 9, 2012

\begin{abstract}
Nitric oxide (NO), is endogenously synthesized from L-arginine by nitric oxide synthase (NOS), exhibits a dual role in sensitivity to radiotherapy and chemotherapy of cancer cells. The aim of this study was to evaluate the influence of polymorphisms in NOS genes on treatment response of non-small-cell lung cancer (NSCLC) patients after radiochemotherapy.

A cohort of 198 NSCLC patients treated with radiochemotherapy between 2009 and 2011 were included in this study. Genotyping analyses of 35 SNPs ( NOS2A, 21 and NOS3, 14) in each sample were conducted by using the Sequenom MassArray system. Unconditional logistic regression was performed to assess the association between treatment response and each genotype while adjusting or not for other covariates.

Of 198 patients, 87 (43.9\%) had objective responses, and 111(56.1\%) did not respond. We observed no significant associations between treatment response and each genotype. While adjusting for other covariates, the associations were also not significant.

Our results suggest that genetic variations within the NOS2A and NOS3 genes may not influence the treatment response in NSCLC patients with radiochemotherapy. Future studies in this problem are required to confirm our findings.
\end{abstract}

Key words: single nucleotide polymorphism, nitric oxide synthase, non-small cell lung cancer, radiochemotherapy, treatment response

Worldwide, lung cancer is the most common cause of cancer-related death in men and women, and is responsible for 1.4 million deaths annually, as of 2008 [1]. Most patients diagnosed with non-small cell lung cancer (NSCLC) presented with advanced disease. For these patients, chemotherapy and radiotherapy were often the primary choices of treatment. Although platinum-based chemotherapy alone or in combination with radiotherapy is effective in treating some patients, insensitivity to radiochemotherapy is still a major problem in cancer treatment [2] and usually predicted shorter overall survival [3]. The basis behind treatment resistance either as primary or secondary, i.e., occurring after an initial treatment response, is still challenging to understand [4]. In previous studies, genetic factors were proved to influence the effectiveness of lung cancer treatment [5-14].
Nitric oxide (NO), a reactive radical, is endogenously synthesized from L-arginine by nitric oxide synthase (NOS), which exists as three isoforms: neuronal NOS (nNOS/NOS1), inducible NOS (iNOS/NOS2), endothelial NOS (eNOS/ NOS3) [15]. The nNOS and eNOS isoforms are constitutively expressed in a variety of cell types including the endothelium, platelets, and neurons [16]. However, iNOS is absent in resting cells, but is capable of being rapidly expressed in response to proinflammatory stimuli such as cytokines and the HIF-1 mediated pathway [17]. In cancer biology and treatment, NO may have dual effects [18], cytoprotective and cytotoxic, which depended upon NO concentrations [19] or the specific gene, such as P53 [20]. On the one hand, NO is considered as an efficient hypoxic radiosensitizer [21-27] or a chemosensitizer [28-30]. Further studies suggested that 
iNOS overexpression by adenoviral gene delivery enhances the radioresponsiveness of colorectal cancer via p53 activation and caspase-dependent apoptotic mechanism [30-33]. On the other hand, NO radicals could contribute to the induction of radioresistance [34] and chemotherapeutic resistance $[35,36]$. In lung cancer patients, several cytokines (IL-1 $\beta$, IL-6, IFN- $\gamma$, TNF- $\alpha$, TGF- $\beta$, etc.) are produced to enhance the production of NO [37]. These studies suggest that NO and NOS may be a novel therapeutic strategy for NSCLC treatment.

Given the uncertainty between NOS and treatment response, we speculated that genetic variants in NOS genes may alter their expression, activity or functions and in turn influence the effects of cancer treatment. To test this hypothesis, we genotyped 198 specimens collected from a cohort of NSCLC patients for 35 single nucleotide polymorphisms (SNPs) in NOS2A and NOS3 genes by using the Sequenom MassArray system, and then evaluated their associations with radiochemotherapy sensitivity. The result is expected to guide individualized NSCLC therapy.

\section{Patients and Methods}

Patient Population and Clinical Data Collection. In this study, a total of 198 DNA samples available from newly diagnosed lung cancer patients were prospectively collected between December 2009 and January 2011 at Radiation Oncology Department in Shandong Cancer Hospital (Jinan). All patients were Chinese and were diagnosed with histologically confirmed locally advanced (stage III) or advanced (stage IV) NSCLC, Karnofsky performance status (KPS) $\geq 60$, and an expected survival of $>6$ months. Each patient signed an informed consent and was entered into the clinical research database prior to study entry. This study was approved by Shandong Cancer Hospital institutional review board. The clinical information including sex, age, histology, performance status, smoking status, clinical stage, tumor location, total radiation dose, radiotherapy technique (two- or three-dimension), chemotherapy or other factors were collected. Patient responses to treatment including complete response (CR), partial response (PR), stable disease (SD) and progressive disease (PD) were determined by the Response Evaluation Criteria in Solid Tumors (RECIST) Version 1.1 [38]. For data analysis, CR and PR were combined as responders, and SD and PD were grouped as non-responders.

Radiotherapy. All patients received radiotherapy with 6MV X-rays from linear accelerators (21EX, 23EX or Trilogy; Varian Inc., CA, USA). Target volumes were defined according to the report of International Commission on Radiological Units. The gross tumor volume (GTV) included the primary disease plus any involved regional lymph nodes. The planning target volume (PTV) was considered to include the GTV plus a 10 - to $15-\mathrm{mm}$ margin. $95 \%$ isodose line encompassed the PTV. Planning objective for total lung receiving $>20 \mathrm{~Gy}\left(\mathrm{~V}_{20}\right)$ was limited to $\leq 35 \%$. Treatment planning was optimized using
Philips Pinnacle 3 planning system (Philips Radiation Oncology Systems, Milpitas, CA, USA).

Chemotherapy Regimens. Patients were treated with one of the following regimens: (1) GP regimens $\left(1000 \mathrm{mg} / \mathrm{m}^{2}\right.$ of gemcitabine on day 1 and day 8 , plus $25 \mathrm{mg} / \mathrm{m}^{2}$ of cisplatin on day 1 through day 3 , repeated every 3 weeks); (2) TP regimens $\left(135 \mathrm{mg} / \mathrm{m}^{2}\right.$ of taxol on day 1 , plus $25 \mathrm{mg} / \mathrm{m}^{2}$ of cisplatin on day 1 through day 3 or carboplatin at a dose calculated to produce an area under the serum concentration-time curve of $6.0 \mathrm{~min} \cdot \mathrm{mg} / \mathrm{mL}$, repeated every 3 weeks) or DP regimens (docetaxel $60 \mathrm{mg} / \mathrm{m}^{2}$ followed by cisplatin $25 \mathrm{mg} / \mathrm{m}^{2}$ on day 1 through day 3 , repeated every 3 weeks); (3) NP regimens $\left(25 \mathrm{mg} / \mathrm{m}^{2}\right.$ of vinorelbine on day 1 and day 8 , plus $25 \mathrm{mg} / \mathrm{m}^{2}$ of cisplatin on day 1 through day 3 , repeated every 3 weeks) (4) irinotecan $60 \mathrm{mg} / \mathrm{m}^{2}$ on days 1,8 , and 15 and cisplatin $80 \mathrm{mg} / \mathrm{m}^{2}$ on day 1 , repeated every 4 weeks. Each treatment was repeated for more than two cycles unless the patient met the criteria for PD or experienced unacceptable toxicity. Chemotherapy dosage was modified by toxicities in subsequent courses.

SNP Selection. We chose tag SNPs according to a similar approach in the previous article [39]. Tag SNPs were selected based on the ability to tag surrounding variants (iNOS, chr17 and eNOS, chr7) in the Han Chinese panel (Beijing, China) of the International HapMap project, NCBI build B36 assembly HapMap phase II+III (http://www.hapmap.org). The region used in tagSNP selection included a region from 3,000 bp 5 ' upstream to $1000 \mathrm{bp} 3^{\prime}$ downstream of NOS2A or NOS3. Tagger software included in Haploview software 4.2 was used to select tag SNPs. The pairwise algorithm of the Tagger program was used to select the tags. An $\mathrm{r}^{2}$ of 0.8 was defined as the coefficient threshold for tag selection and minor allele frequency (MAF) was 0.05 [40]. In addition, common SNPs based on previous report of other diseases in NOS were included in this study. The characteristics of 35 selected SNPs were listed in Table 1.

DNA Collection and Genotyping. A $1 \mathrm{~mL}$ whole-blood sample was obtained from each patient before treatment. The blood samples were collected in EDTA vacutainer tubes and stored at $-80^{\circ} \mathrm{C}$ until analysis. Genomic DNA was extracted from whole-blood cells using AxyPrep Blood Genomic DNA Miniprep Kit (Axygen, USA) according to the manufacturer's instructions. The DNA purity and concentration were determined by spectro- photometric measurement of absorbance at 260 and $280 \mathrm{~nm}$, respectively. Approximately $25 \mathrm{ng}$ of genomic DNA was used to genotype each sample using the Sequenom MassArray system (San Diego, USA). The sample DNA was amplified by multiplex PCR reaction and the PCR products were then used for single-base extension reaction. The resulting products were desalted and transferred to a 384element SpectroCHIP array. Allele detection was performed using MALDI-TOF MS. The mass spectrograms were analysed by the Sequenom MassARRAY TYPER software (San Diego, USA). The whole SNPs genotyping was conducted at Shandong Provincial Key Lab for Dermatovenereology (Jinan, China). 
Table 1. Characteristics of 35 SNPs in NOS2A and NOS3

\begin{tabular}{|c|c|c|c|c|c|c|}
\hline Gene & SNP ID & Chromosome & Position & Allele & Domain & \\
\hline NOS3 & rs7830 & 7 & 150340504 & $\mathrm{~A} / \mathrm{C}$ & Intron & $\operatorname{tagSNP}$ \\
\hline NOS3 & rs743507 & 7 & 150338421 & $\mathrm{~A} / \mathrm{G}$ & Intron & tagSNP \\
\hline NOS3 & rs1800781 & 7 & 150323377 & $\mathrm{~A} / \mathrm{G}$ & Intron & tagSNP \\
\hline NOS3 & rs3918188 & 7 & 150333714 & $\mathrm{~A} / \mathrm{C}$ & Intron & tagSNP \\
\hline NOS3 & rs3918227 & 7 & 150331879 & $\mathrm{~A} / \mathrm{C}$ & Intron & tagSNP \\
\hline NOS3 & rs891512 & 7 & 150339022 & $\mathrm{~A} / \mathrm{G}$ & Intron & tagSNP \\
\hline NOS3 & rs11771443 & 7 & 150318620 & $\mathrm{C} / \mathrm{T}$ & Promoter & tagSNP \\
\hline NOS2A & rs2297515 & 17 & 23117460 & $\mathrm{~A} / \mathrm{C}$ & Intron & $\operatorname{tagSNP}$ \\
\hline NOS2A & rs2297518 & 17 & 23120724 & $\mathrm{~A} / \mathrm{G}$ & CDS-nonsynonymous & tagSNP \\
\hline NOS2A & rs2779248 & 17 & 23151959 & $\mathrm{C} / \mathrm{T}$ & Promoter & tagSNP \\
\hline NOS2A & rs3794763 & 17 & 23135353 & $\mathrm{~A} / \mathrm{G}$ & Intron & tagSNP \\
\hline NOS2A & rs4795067 & 17 & 23130802 & $\mathrm{~A} / \mathrm{G}$ & Intron & tagSNP \\
\hline NOS2A & rs8072199 & 17 & 23140975 & $\mathrm{C} / \mathrm{T}$ & Intron & tagSNP \\
\hline NOS2A & rs2314809 & 17 & 23119505 & $\mathrm{C} / \mathrm{T}$ & Intron & tagSNP \\
\hline NOS2A & rs7208775 & 17 & 23109235 & $\mathrm{C} / \mathrm{G}$ & Intron & tagSNP \\
\hline NOS2A & rs16949 & 17 & 23148826 & $\mathrm{C} / \mathrm{T}$ & Intron & $\operatorname{tagSNP}$ \\
\hline NOS2A & rs3730013 & 17 & 23150045 & $\mathrm{C} / \mathrm{T}$ & Intron & tagSNP \\
\hline NOS2A & rs9906835 & 17 & 23113501 & $\mathrm{~A} / \mathrm{G}$ & Intron & $\operatorname{tagSNP}$ \\
\hline NOS2A & rs11080344 & 17 & 23128638 & $\mathrm{C} / \mathrm{T}$ & Intron & tagSNP \\
\hline NOS3 & rs1549758 & 7 & 150326659 & $\mathrm{C} / \mathrm{T}$ & CDS-synonymous & \\
\hline NOS3 & rs1799983 & 7 & 150327044 & $\mathrm{G} / \mathrm{T}$ & CDS-nonsynonymous & \\
\hline NOS3 & rs1800779 & 7 & 150320876 & $\mathrm{~A} / \mathrm{T}$ & Intron & \\
\hline NOS3 & rs 1800780 & 7 & 150329812 & $\mathrm{~A} / \mathrm{G}$ & Intron & \\
\hline NOS3 & rs1800783 & 7 & 150320330 & $\mathrm{~A} / \mathrm{T}$ & Intron & \\
\hline NOS3 & rs3918174 & 7 & 150328227 & $\mathrm{~A} / \mathrm{G}$ & Intron & \\
\hline NOS3 & rs4496877 & 7 & 150311439 & $\mathrm{G} / \mathrm{T}$ & 5'UTR & \\
\hline NOS2A & rs944722 & 17 & 23116164 & $\mathrm{C} / \mathrm{T}$ & Intron & \\
\hline NOS2A & rs 944725 & 17 & 23133698 & $\mathrm{C} / \mathrm{T}$ & Intron & \\
\hline NOS2A & rs1137933 & 17 & 23130059 & $\mathrm{C} / \mathrm{T}$ & CDS-synonymous & \\
\hline NOS2A & rs2072324 & 17 & 23141023 & $\mathrm{~A} / \mathrm{C}$ & Intron & \\
\hline NOS2A & rs2248814 & 17 & 23124448 & $\mathrm{~A} / \mathrm{G}$ & Intron & \\
\hline NOS2A & rs2255929 & 17 & 23112094 & $\mathrm{~A} / \mathrm{T}$ & Intron & \\
\hline NOS2A & rs2314810 & 17 & 23128237 & $\mathrm{C} / \mathrm{G}$ & Intron & \\
\hline NOS2A & rs 10459953 & 17 & 23151645 & $\mathrm{C} / \mathrm{G}$ & 5'UTR & \\
\hline NOS2A & rs17722851 & 17 & 23134963 & $\mathrm{~A} / \mathrm{T}$ & Intron & \\
\hline
\end{tabular}

Abbreviations: SNP, single nucleotide polymorphism; 5'UTR, 5’ untranslational region; CDS, Coding Sequence.

Statistical Analysis. The frequencies of different genotypes were compared between patients with and without treatment response through the Chi-square test. In multivariate analyses, unconditional logistic regression was performed to assess the association between treatment response and each genotype while adjusting for other covariates, including radiation dose, sex, age, histology, performance status, smoking status, clinical stage, tumor location, chemo-radiotherapy type. In the regression analysis, the dependent variable was patient response to treatment; patients who did not respond to treatment (SD + $\mathrm{PD}$ ) were compared to patients who responded to treatment
$(\mathrm{CR}+\mathrm{PR})$. Odds ratios (OR) and their corresponding $95 \%$ confidence intervals (CI) were calculated using the logistic regression model. $P$ value $<0.05$ was considered statistically significant. All tests were two-sided. Statistical analysis was performed using IBM SPSS statistical version 19.0 software.

\section{Results}

Clinical Characteristics. Table 2 shows the baseline clinical characteristics of the 198 NSCLC patients (150 men and 48 women; median age, 60 years; range, 25 to 87 years), of whom 
Table 2. Baseline Clinical Characteristics of Patients $(n=198)$

\begin{tabular}{|c|c|c|}
\hline Characteristics & No. & $\%$ \\
\hline \multicolumn{3}{|l|}{ Sex } \\
\hline Female & 48 & 24.2 \\
\hline Male & 150 & 75.8 \\
\hline \multicolumn{3}{|l|}{ KPS } \\
\hline $90-100$ & 105 & 53.0 \\
\hline 80 & 66 & 33.3 \\
\hline$<80$ & 27 & 13.6 \\
\hline \multicolumn{3}{|l|}{ Histopathology } \\
\hline Squamous cell & 78 & 39.4 \\
\hline Adenocarcinoma & 58 & 29.3 \\
\hline NSCLC, NOS & 62 & 31.3 \\
\hline \multicolumn{3}{|l|}{ Tumor location } \\
\hline Peripheral & 128 & 64.6 \\
\hline Central & 70 & 35.4 \\
\hline \multicolumn{3}{|l|}{ Clinical stage } \\
\hline III & 106 & 53.5 \\
\hline IV & 92 & 46.5 \\
\hline \multicolumn{3}{|l|}{ Smoking status } \\
\hline Nonsmoker & 48 & 24.2 \\
\hline $\mathrm{CSI} \geq 600$ & 89 & 44.9 \\
\hline $\mathrm{CSI}<600$ & 61 & 30.8 \\
\hline \multicolumn{3}{|l|}{ Treatment } \\
\hline Concurrent chemoradiation & 94 & 47.5 \\
\hline Sequential chemoradiation & 104 & 52.5 \\
\hline \multicolumn{3}{|l|}{ Radiation technique } \\
\hline 2-dimensional radiotherapy & 25 & 12.6 \\
\hline 3-dimensional radiotherapy & 123 & 57.1 \\
\hline IMRT & 60 & 30.3 \\
\hline
\end{tabular}

Abbreviations: KPS, karnofsky performance status; NSCLC, NOS, non-smallcell lung carcinoma, not otherwise specified; CSI, cigarette-smoke index; IMRT, intensity-modulated radiation therapy.

$54.5 \%$ had stage III disease, and $75.8 \%$ were cigarette smokers. Histological types included squamous cell carcinoma (39.4\%), adenocarcinoma (29.3\%), and large cell or not otherwise specified (31.3\%). The median radiation dose was $60.0 \mathrm{~Gy}$ (range, 45 to $80.6 \mathrm{~Gy}) ; 83.3 \%(\mathrm{n}=165)$ of patients received 50 to $70 \mathrm{~Gy}$. Of the 198 patients, $100 \%$ received platinum-based chemotherapy, $94(47.5 \%)$ were given concurrent chemoradiation, and 104 (52.5\%) had sequential chemoradiation. Seventy-four (37.4\%) patients were given GP regimens, 70 (35.4\%) received TP regimens, $40(20.2 \%)$ had NP regimens, and $14(7.1 \%)$ were treated with other combinations. All patients were treated for three to six cycles, with a median of four cycles.

Patient Response to Treatment. Patient response to treatment was evaluated 1 to 2 months after completion of treatment. For 198 patients, 87 (43.9\%) had objective responses $(\mathrm{CR}+\mathrm{PR})$, and $111(56.1 \%)$ had not (SD + PD). For patients who received sequential chemoradiation, 43 (41.3\%) had treatment responses and 61 (58.7\%) showed no response. More patients treated with concurrent chemoradiation had higher response rates $(46.8 \%)$, but the difference between the two groups was not significant $(P=0.439)$. Patients with radio- therapy dose ( $\geq 60$ Gy) was shown to have a higher response than those treated with lower ones $(53.8 \%$ versus $29.6 \%, P=$ 0.001 ). The response rates among all the treatment groups with different chemotherapy regimens were not significantly different $(P=0.221)$. The response rate to treatment for the patients with stage III NSCLC was higher than those with stage IV, but the difference was not statistically significant $(49.1 \%$ versus $38 \%, P=0.119)$. Associations between clinical characteristics and treatment response were shown in Table 3.

Genotypes and Treatment Responses. Successful genotype call rates in 35 SNPs were $97-100 \%$ and no SNP was excluded from analysis. Genotype distributions of 35 SNPs in NOS3 and NOS2A genes and association analysis results were listed in Table 4, Table 5, respectively. We observed no significant associations between treatment response and each genotype. It was noted that treatment responders and non-responders differed obviously with respect to radiation dose. While adjusting for covariates in the multiple logistic regression analysis, the associations were also not significant.

\section{Discussion}

The aim of this study is to investigate the association between genetic variants of NOS and treatment response. Our results suggest that single nucleotide polymorphisms in NOS2A and NOS3 genes are not associated with treatment response of NSCLC patients following radiochemotherapy. Even after adjusting for other covariates, no significant associations were found. On one hand, this negative result was possibly due to: (i) There was a small cohort of patients analyzed. Whenever a negative association study is reported, the power of the study is normally questioned. On this regard we speculate that smaller sample size may be a limiting factor for this study especially for the analysis of treatment effects where we are comparing 87 responders against 111 non-responders. (ii) The role of genetic variants of NOS2A and NOS3 on treatment response may be covered by other clinical or dosimetric factors, such as stage, chemotherapy regimens, radiation dose, etc. On the other hand, since NO and NOS might have totally diverse biological effects on chemoradiotherapy and the evidence in cancer is also conflicting, the hypothesis that NOS gene is responsible for the differences in treatment response of NSCLC patients may be incorrect. In view of the two possible aspects, the large sample validation study is necessary in the future.

In previous studies, genetic factors had been proved to influence the effectiveness of lung cancer treatment [5-14]. Over the past few years, associations between NOS polymorphisms and clinical prognosis of patients with breast cancer, vulvar cancer and NSCLC had also been reported [14, 41-43]. The polymorphism of the 27-bp variable number of tandem repeats (VNTR) in intron 4, not G894T in exon 7 (rs1799983) of the eNOS gene was found to be an independent prognostic factor for survival in advanced stage NSCLC patients treated with standard platinum-based chemotherapy [14]. However, the 
Table 3. Associations between clinical characteristics and treatment response

\begin{tabular}{|c|c|c|c|c|c|c|}
\hline Parameter & Crude OR & $95 \% \mathrm{CI}$ & $P$ value & Adjusted OR & $95 \%$ CI & $P$ value \\
\hline \multicolumn{7}{|l|}{ Sex } \\
\hline Female & 1.000 & & & 1.000 & & \\
\hline Male & 0.904 & $0.47-1.74$ & 0.761 & 0.915 & $0.45-1.90$ & 0.861 \\
\hline Age, years & & & . & & & \\
\hline$<65$ & 1.000 & & & 1.000 & & \\
\hline$\geq 65$ & 1.033 & $0.58-1.85$ & 0.914 & 0.904 & $0.46-1.79$ & 0.773 \\
\hline \multicolumn{7}{|l|}{ KPS } \\
\hline $90-100$ & 1.000 & & & 1.000 & & \\
\hline 80 & 0.646 & $0.35-1.21$ & 0.172 & 0.632 & $0.31-1.29$ & 0.208 \\
\hline$<80$ & 0.728 & $0.31-1.71$ & 0.468 & 0.684 & $0.27-1.76$ & 0.431 \\
\hline \multicolumn{7}{|l|}{ Stage } \\
\hline IV & 1.000 & & & 1.000 & & \\
\hline III & 1.568 & $0.90-2.77$ & 0.120 & 1.083 & $0.56-2.02$ & 0.816 \\
\hline \multicolumn{7}{|l|}{ Hypertension } \\
\hline No & 1.000 & & & 1.000 & & \\
\hline Yes & 2.036 & $0.98-4.24$ & 0.057 & 2.459 & $0.98-6.16$ & 0.055 \\
\hline \multicolumn{7}{|l|}{ COPD } \\
\hline No & 1.000 & & & 1.000 & & \\
\hline Yes & 1.324 & $0.58-3.03$ & 0.505 & 1.476 & $0.56-3.92$ & 0.434 \\
\hline \multicolumn{7}{|l|}{ Tumor location } \\
\hline Central & 1.000 & & & 1.000 & & \\
\hline Peripheral & 0.626 & $0.35-1.13$ & 0.117 & 0.766 & $0.40-1.52$ & 0.446 \\
\hline \multicolumn{7}{|l|}{ Histology } \\
\hline Squamous cell & 1.000 & & & 1.000 & & \\
\hline Adenocarcinoma & 0.743 & $0.37-1.48$ & 0.396 & 1.031 & $0.47-2.30$ & 0.941 \\
\hline NSCLC, NOS & 0.711 & $0.36-1.40$ & 0.322 & 0.747 & $0.35-1.60$ & 0.451 \\
\hline \multicolumn{7}{|l|}{ Tobacco use } \\
\hline Never & 1.000 & & & 1.000 & & \\
\hline $\mathrm{CSI}<600$ & 0.922 & $0.46-1.90$ & 0.821 & 0.922 & $0.43-1.98$ & 0.835 \\
\hline $\mathrm{CSI} \geq 600$ & 0.878 & $0.41-1.88$ & 0.738 & 0.843 & $0.36-2.00$ & 0.693 \\
\hline \multicolumn{7}{|l|}{ CRT } \\
\hline Yes & 1.000 & & & 1.000 & & \\
\hline No & 0.801 & $0.46-1.41$ & 0.440 & 0.812 & $0.44-1.51$ & 0.512 \\
\hline \multicolumn{7}{|l|}{ Radiation dose, Gy } \\
\hline$<60$ & 1.000 & & & 1.000 & & \\
\hline$\geq 60$ & 2.771 & $1.52-5.10$ & 0.001 & 2.601 & $1.35-5.00$ & 0.004 \\
\hline
\end{tabular}

Abbreviations: OR, odds ratios; 95\%CI, 95\% confidence intervals; KPS, karnofsky performance status; COPD, chronic obstructive pulmonary disease; NSCLC, NOS, non-small-cell lung carcinoma, not otherwise specified; CSI, cigarette-smoke index; CRT, concurrent chemoradiation.

author continued to analyze the treatment response among patients without definitive thoracic radiotherapy, indicating that the effect of the polymorphism on survival was not associated with treatment response [14]. The present study, revealed that there was no association between rs1799983 of the eNOS gene and treatment response in NSCLC patients, was consistent with this study.

Strength of this study is the inclusion of a considerable number of SNPs for which an association with cancer is biologically plausible and/or has been previously reported. However, our study has several limitations. Firstly, because we selected the most informative SNPs with high MAF and $\mathrm{r}^{2}$, some causal variants might be missed. Secondly, the single-institutional study design contains the potential to introduce a selection bias. Thirdly, the analysis based upon a small number of enrolled patients could potentially lead to false-negative results. Finally, because all patients in our study were Han Chinese, it is uncertain whether these 
Table 4. Genotypes in NOS3 and its association with treatment response for NSCLC patients

\begin{tabular}{|c|c|c|c|c|c|c|c|c|}
\hline \multirow[t]{2}{*}{ SNPs } & \multirow[t]{2}{*}{ Genotype } & \multicolumn{2}{|c|}{$\mathrm{CR}+\mathrm{PR}$} & \multicolumn{2}{|c|}{$\mathrm{SD}+\mathrm{PD}$} & \multirow{2}{*}{$\begin{array}{c}\text { Adjusted } \\
\text { OR }\end{array}$} & \multirow[t]{2}{*}{$95 \%$ CI } & \multirow{2}{*}{$\begin{array}{c}\text { Adjusted } \\
\mathrm{P} \text { value }\end{array}$} \\
\hline & & $\mathrm{N}$ & $\%$ & $\mathrm{~N}$ & $\%$ & & & \\
\hline rs11771443 & $\mathrm{CC}$ & 31 & 36.0 & 42 & 39.3 & 1 & & \\
\hline \multirow[t]{2}{*}{$(\mathrm{n}=193)$} & $\mathrm{CT}$ & 41 & 47.7 & 43 & 40.2 & 1.419 & $0.71-2.83$ & 0.320 \\
\hline & $\mathrm{TT}$ & 14 & 16.3 & 22 & 20.6 & 0.863 & $0.40-2.11$ & 0.746 \\
\hline rs1800779 & $\mathrm{AA}$ & 73 & 84.9 & 93 & 83.8 & 1 & & \\
\hline \multirow[t]{2}{*}{$(\mathrm{n}=197)$} & $A G$ & 11 & 12.8 & 15 & 13.5 & 0.898 & $0.40-2.30$ & 0.817 \\
\hline & GG & 2 & 2.3 & 3 & 2.7 & 0.901 & $0.12-6.60$ & 0.918 \\
\hline rs1800781 & AA & 1 & 1.1 & 3 & 2.7 & 1 & & \\
\hline \multirow[t]{2}{*}{$(\mathrm{n}=197)$} & $\mathrm{AG}$ & 12 & 13.8 & 14 & 12.7 & 2.580 & $0.18-36.50$ & 0.483 \\
\hline & GG & 74 & 85.1 & 93 & 84.5 & 2.531 & $0.21-31.10$ & 0.468 \\
\hline rs1799983 & GG & 70 & 81.4 & 85 & 77.3 & 1 & & \\
\hline \multirow[t]{2}{*}{$(\mathrm{n}=196)$} & GT & 16 & 18.6 & 23 & 20.9 & 0.900 & $0.42-1.94$ & 0.788 \\
\hline & $\mathrm{TT}$ & 0 & 0 & 2 & 1.8 & 0.000 & $0.00-$ & 0.999 \\
\hline rs3918174 & $\mathrm{AA}$ & 74 & 86.0 & 90 & 84.1 & 1 & & \\
\hline \multirow[t]{2}{*}{$(\mathrm{n}=193)$} & $A G$ & 11 & 12.8 & 14 & 13.1 & 1.040 & $0.41-2.64$ & 0.934 \\
\hline & GG & 1 & 1.2 & 3 & 2.8 & 0.402 & $0.03-4.91$ & 0.475 \\
\hline rs3918227 & AA & 0 & 0 & 1 & 0.9 & 1 & & \\
\hline \multirow[t]{2}{*}{$(\mathrm{n}=192)$} & $\mathrm{AC}$ & 9 & 10.8 & 12 & 11.0 & $5.638 \mathrm{E} 8$ & $0.00-$ & 1.000 \\
\hline & $\mathrm{CC}$ & 74 & 89.2 & 96 & 88.1 & $5.637 \mathrm{E} 8$ & $0.00-$ & 1.000 \\
\hline rs891512 & $\mathrm{AG}$ & 4 & 4.7 & 11 & 10.0 & 1 & & \\
\hline$(\mathrm{n}=196)$ & GG & 82 & 95.3 & 99 & 90.0 & 1.913 & $0.53-6.92$ & 0.323 \\
\hline rs7830 & AA & 15 & 17.2 & 19 & 17.3 & 1 & & \\
\hline \multirow[t]{2}{*}{$(\mathrm{n}=197)$} & $\mathrm{AC}$ & 36 & 41.4 & 54 & 49.1 & 0.915 & $0.40-2.21$ & 0.844 \\
\hline & $\mathrm{CC}$ & 36 & 41.4 & 37 & 33.6 & 1.635 & $0.70-4.01$ & 0.283 \\
\hline rs4496877 & GG & 74 & 85.1 & 92 & 83.6 & 1 & & \\
\hline \multirow[t]{2}{*}{$(\mathrm{n}=195)$} & GT & 12 & 13.8 & 15 & 13.6 & 0.964 & $0.40-2.40$ & 0.937 \\
\hline & $\mathrm{TT}$ & 1 & 1.1 & 3 & 2.7 & 0.394 & $0.03-4.83$ & 0.466 \\
\hline rs1800783 & AA & 2 & 2.3 & 3 & 2.7 & 1 & & \\
\hline \multirow[t]{2}{*}{$(\mathrm{n}=197)$} & $\mathrm{AT}$ & 11 & 12.6 & 15 & 13.6 & 1.001 & $0.12-8.64$ & 1.000 \\
\hline & $\mathrm{TT}$ & 74 & 85.1 & 92 & 83.6 & 1.156 & $0.16-8.40$ & 0.886 \\
\hline rs1549758 & $\mathrm{CC}$ & 61 & 70.1 & 75 & 67.6 & 1 & & \\
\hline \multirow[t]{2}{*}{$(\mathrm{n}=198)$} & CT & 24 & 27.6 & 31 & 27.9 & 0.840 & $0.42-1.70$ & 0.622 \\
\hline & $\mathrm{TT}$ & 2 & 2.3 & 5 & 4.5 & 0.515 & $0.10-2.90$ & 0.447 \\
\hline rs1800780 & AA & 10 & 11.5 & 11 & 9.9 & 1 & & \\
\hline \multirow[t]{2}{*}{$(\mathrm{n}=198)$} & $\mathrm{AG}$ & 38 & 43.7 & 57 & 51.4 & 0.695 & $0.25-1.95$ & 0.489 \\
\hline & GG & 39 & 44.8 & 43 & 38.7 & 0.887 & $0.31-2.54$ & 0.824 \\
\hline rs3918188 & AA & 11 & 12.6 & 10 & 9.0 & 1 & & \\
\hline \multirow[t]{2}{*}{$(\mathrm{n}=198)$} & $\mathrm{AC}$ & 32 & 36.8 & 57 & 51.4 & 0.442 & $0.15-1.27$ & 0.130 \\
\hline & $\mathrm{CC}$ & 44 & 50.6 & 44 & 39.6 & 0.786 & $0.28-2.20$ & 0.643 \\
\hline rs743507 & $\mathrm{AA}$ & 40 & 40.6 & 60 & 54.1 & 1 & & \\
\hline \multirow[t]{2}{*}{$(\mathrm{n}=198)$} & AG & 42 & 48.3 & 49 & 44.1 & 1.458 & $0.78-2.74$ & 0.240 \\
\hline & GG & 5 & 5.7 & 2 & 1.8 & 3.730 & $0.60-23.12$ & 0.157 \\
\hline
\end{tabular}

Abbreviations: NSCLC, non-small-cell lung carcinoma; SNPs, single nucleotide polymorphisms; CR, complete response; PR, partial response; SD, stable disease; PD, progressive disease.

results can be generalized to other populations. Because ethnic differences in the genotypes in NOS genes may exist, other studies in different population are required to confirm such results.

\section{Conclusions}

To our knowledge, this is the comprehensive report investigating the association between genetic polymorphisms of 
Table 5. Genotypes in NOS2A and its association with treatment response for NSCLC patients

\begin{tabular}{|c|c|c|c|c|c|c|c|c|}
\hline \multirow[t]{2}{*}{ SNP } & \multirow[t]{2}{*}{ Genotype } & \multicolumn{2}{|c|}{$\mathrm{CR}+\mathrm{PR}$} & \multicolumn{2}{|c|}{$\mathrm{SD}+\mathrm{PD}$} & \multirow{2}{*}{$\begin{array}{c}\text { Adjusted } \\
\text { OR }\end{array}$} & \multirow[t]{2}{*}{$95 \%$ CI } & \multirow{2}{*}{$\begin{array}{l}\text { Adjusted } \\
P \text { value }\end{array}$} \\
\hline & & $\mathrm{N}$ & $\%$ & $\mathrm{~N}$ & $\%$ & & & \\
\hline rs2255929 & $\mathrm{AA}$ & 50 & 57.5 & 59 & 53.6 & 1 & & \\
\hline \multirow[t]{2}{*}{$(\mathrm{n}=197)$} & $\mathrm{AT}$ & 32 & 36.8 & 44 & 40.0 & 0.750 & $0.39-1.44$ & 0.384 \\
\hline & $\mathrm{TT}$ & 5 & 5.7 & 7 & 6.4 & 0.876 & $0.23-3.29$ & 0.844 \\
\hline rs9906835 & $\mathrm{AA}$ & 26 & 30.2 & 38 & 35.2 & 1 & & \\
\hline \multirow[t]{2}{*}{$(\mathrm{n}=194)$} & AG & 40 & 46.5 & 48 & 44.4 & 1.324 & $0.65-2.68$ & 0.436 \\
\hline & GG & 20 & 23.3 & 22 & 20.4 & 1.269 & $0.54-3.00$ & 0.582 \\
\hline rs944722 & $\mathrm{CC}$ & 4 & 4.7 & 5 & 4.7 & 1 & & \\
\hline \multirow[t]{2}{*}{$(\mathrm{n}=193)$} & $\mathrm{CT}$ & 32 & 37.2 & 40 & 37.4 & 0.632 & $0.14-2.81$ & 0.547 \\
\hline & $\mathrm{TT}$ & 50 & 58.1 & 62 & 57.9 & 0.761 & $0.18-3.30$ & 0.715 \\
\hline rs2248814 & AA & 6 & 7.0 & 9 & 8.4 & 1 & & \\
\hline \multirow[t]{2}{*}{$(\mathrm{n}=193)$} & AG & 32 & 37.2 & 36 & 33.6 & 1.195 & $0.36-3.95$ & 0.770 \\
\hline & GG & 48 & 55.8 & 62 & 57.9 & 1.164 & $0.36-3.80$ & 0.799 \\
\hline rs11080344 & $\mathrm{CC}$ & 34 & 39.1 & 54 & 49.1 & 1 & & \\
\hline \multirow[t]{2}{*}{$(\mathrm{n}=197)$} & CT & 41 & 47.1 & 46 & 41.8 & 1.221 & $0.62-2.40$ & 0.561 \\
\hline & $\mathrm{TT}$ & 12 & 13.8 & 10 & 9.1 & 2.011 & $0.70-5.80$ & 0.194 \\
\hline rs4795067 & $\mathrm{AA}$ & 58 & 67.4 & 67 & 64.1 & 1 & & \\
\hline \multirow[t]{2}{*}{$(n=195)$} & AG & 24 & 27.9 & 37 & 31.3 & 0.922 & $0.47-1.83$ & 0.815 \\
\hline & GG & 4 & 4.7 & 5 & 4.6 & 1.192 & $0.25-5.61$ & 0.824 \\
\hline rs944725 & $\mathrm{CC}$ & 31 & 36.0 & 46 & 42.2 & 1 & & \\
\hline \multirow[t]{2}{*}{$(\mathrm{n}=195)$} & $\mathrm{CT}$ & 42 & 48.8 & 47 & 43.1 & 1.471 & $0.75-2.87$ & 0.258 \\
\hline & $\mathrm{TT}$ & 13 & 15.1 & 16 & 14.7 & 1.269 & $0.47-3.41$ & 0.637 \\
\hline rs17722851 & AT & 6 & 7.0 & 11 & 10.1 & 1 & & \\
\hline$(n=195)$ & $\mathrm{TT}$ & 80 & 93.0 & 98 & 89.9 & 1.824 & $0.60-5.60$ & 0.294 \\
\hline rs8072199 & $\mathrm{CC}$ & 68 & 79.1 & 92 & 84.4 & 1 & & \\
\hline \multirow[t]{2}{*}{$(\mathrm{n}=195)$} & $\mathrm{CT}$ & 16 & 18.6 & 17 & 15.6 & 1.387 & $0.60-3.20$ & 0.443 \\
\hline & TT & 2 & 2.3 & 0 & 0 & $4.596 \mathrm{E} 9$ & $0.00-$ & 0.999 \\
\hline rs3730013 & $\mathrm{CC}$ & 30 & 34.9 & 38 & 35.5 & 1 & & \\
\hline \multirow[t]{2}{*}{$(\mathrm{n}=193)$} & $\mathrm{CT}$ & 41 & 47.7 & 48 & 44.9 & 1.148 & $0.57-2.31$ & 0.700 \\
\hline & $\mathrm{TT}$ & 15 & 17.4 & 21 & 19.6 & 0.935 & $0.38-2.33$ & 0.885 \\
\hline rs10459953 & $\mathrm{CC}$ & 16 & 18.4 & 24 & 22.2 & 1 & & \\
\hline \multirow[t]{2}{*}{$(\mathrm{n}=195)$} & CG & 39 & 44.8 & 50 & 46.3 & 1.384 & $0.60-3.21$ & 0.448 \\
\hline & GG & 32 & 36.8 & 34 & 31.5 & 1.874 & $0.77-4.55$ & 0.165 \\
\hline rs7208775 & CG & 84 & 96.6 & 107 & 97.3 & 1 & & \\
\hline$(\mathrm{n}=197)$ & GG & 3 & 3.4 & 3 & 2.7 & 1.219 & $0.22-6.73$ & 0.820 \\
\hline rs2297515 & $\mathrm{AA}$ & 66 & 75.9 & 94 & 84.7 & 1 & & \\
\hline$(n=198)$ & $\mathrm{AC}$ & 21 & 24.1 & 17 & 15.3 & 1.885 & $0.88-4.06$ & 0.105 \\
\hline rs2314809 & $\mathrm{CC}$ & 4 & 4.6 & 5 & 4.5 & 1 & & \\
\hline \multirow[t]{2}{*}{$(\mathrm{n}=198)$} & $\mathrm{CT}$ & 32 & 36.8 & 43 & 38.7 & 0.573 & $0.13-2.55$ & 0.465 \\
\hline & $\mathrm{TT}$ & 51 & 58.6 & 63 & 56.8 & 0.745 & $0.17-3.23$ & 0.694 \\
\hline rs2297518 & AA & 4 & 4.6 & 3 & 2.7 & 1 & & \\
\hline \multirow[t]{2}{*}{$(\mathrm{n}=197)$} & AG & 15 & 17.2 & 25 & 22.7 & 0.428 & $0.07-2.74$ & 0.371 \\
\hline & GG & 68 & 78.2 & 82 & 74.5 & 0.489 & $0.09-2.77$ & 0.418 \\
\hline rs2314810 & $\mathrm{CC}$ & 5 & 5.7 & 6 & 5.4 & 1 & & \\
\hline \multirow[t]{2}{*}{$(\mathrm{n}=198)$} & CG & 29 & 33.3 & 38 & 34.2 & 0.720 & $0.18-2.92$ & 0.646 \\
\hline & GG & 53 & 60.9 & 67 & 60.4 & 0.850 & $0.22-3.36$ & 0.816 \\
\hline rs1137933 & $\mathrm{CC}$ & 69 & 79.3 & 80 & 72.1 & 1 & & \\
\hline$(\mathrm{n}=198)$ & CT & 17 & 19.5 & 30 & 27.0 & 0.898 & $0.42-1.93$ & 0.783 \\
\hline & $\mathrm{TT}$ & 1 & 1.1 & 1 & 0.9 & 0.670 & $0.03-15.90$ & 0.804 \\
\hline
\end{tabular}


Table 5. Continued

\begin{tabular}{|c|c|c|c|c|c|c|c|c|}
\hline \multirow[t]{2}{*}{ SNP } & \multirow[t]{2}{*}{ Genotype } & \multicolumn{2}{|c|}{$\mathrm{CR}+\mathrm{PR}$} & \multicolumn{2}{|c|}{$\mathrm{SD}+\mathrm{PD}$} & \multirow{2}{*}{$\begin{array}{c}\text { Adjusted } \\
\text { OR }\end{array}$} & \multirow[t]{2}{*}{$95 \% \mathrm{CI}$} & \multirow{2}{*}{$\begin{array}{c}\text { Adjusted } \\
P \text { value }\end{array}$} \\
\hline & & $\mathrm{N}$ & $\%$ & $\mathrm{~N}$ & $\%$ & & & \\
\hline rs3794763 & AA & 11 & 12.6 & 15 & 13.5 & 1 & & \\
\hline \multirow[t]{2}{*}{$(\mathrm{n}=198)$} & AG & 45 & 51.7 & 44 & 39.6 & 1.438 & $0.55-3.73$ & 0.456 \\
\hline & GG & 31 & 35.6 & 52 & 46.8 & 0.829 & $0.31-2.19$ & 0.706 \\
\hline rs2072324 & $\mathrm{AA}$ & 5 & 5.7 & 12 & 10.8 & 1 & & \\
\hline \multirow[t]{2}{*}{$(\mathrm{n}=198)$} & $\mathrm{AC}$ & 40 & 46.0 & 43 & 38.7 & 2.370 & $0.69-8.14$ & 0.170 \\
\hline & $\mathrm{CC}$ & 42 & 48.3 & 56 & 50.5 & 1.971 & $0.57-6.80$ & 0.283 \\
\hline rs16949 & $\mathrm{CC}$ & 4 & 4.6 & 3 & 2.7 & 1 & & \\
\hline \multirow[t]{2}{*}{$(\mathrm{n}=197)$} & $\mathrm{CT}$ & 19 & 21.8 & 26 & 23.6 & 0.747 & $0.11-4.91$ & 0.761 \\
\hline & $\mathrm{TT}$ & 64 & 73.6 & 81 & 73.6 & 0.585 & $0.10-3.55$ & 0.560 \\
\hline rs2779248 & $\mathrm{TT}$ & 65 & 75.6 & 80 & 72.1 & 1 & & \\
\hline \multirow[t]{2}{*}{$(\mathrm{n}=197)$} & $\mathrm{CT}$ & 21 & 24.4 & 27 & 24.3 & 0.938 & $0.45-1.96$ & 0.865 \\
\hline & $\mathrm{CC}$ & 0 & 0 & 4 & 3.6 & 0.000 & $0.00-$ & 0.999 \\
\hline
\end{tabular}

Abbreviations: NSCLC, non-small-cell lung carcinoma; SNPs, single nucleotide polymorphisms; CR, complete response; PR, partial response; SD, stable disease; PD, progressive disease.

NOS and treatment response. Our results indicate that genetic variations within the NOS2A and NOS3 genes may not influence the treatment response in locally advanced or advanced NSCLC patients with radiochemotherapy.

Acknowledgements: We thank all the patients for giving consent to participate in the study. This work was supported, in part, by the National Nature Science Foundation (Grant No. 30970861) and by Science and Technology Project of Shandong Province (2009GG10002011 and 2011GSF11824).

\section{References}

[1] JEMAL A, BRAY F, CENTER MM, FERLAY J, WARD E, et al. Global cancer statistics. CA Cancer J Clin 2011; 61: 69-90. http: //dx.doi.org/10.3322/caac.20107

[2] SOCINSKI MA. Cytotoxic chemotherapy in advanced non-small cell lung cancer: a review of standard treatment paradigms. Clin Cancer Res 2004; 10: 4210s-4214s. http: //dx.doi.org/10.1158/1078-0432.CCR-040009

[3] D'AMATO TA, PETTIFORD BL, SCHUCHERT MJ, PARKER R, RICKETTS WA, et al. Survival among patients with platinum resistant, locally advanced non-small cell lung cancer treated with platinum-based systemic therapy. Ann Surg Oncol 2009; 16: 2848-2855. http: //dx.doi.org/10.1245/ $\underline{\text { s10434-009-0608-0 }}$

[4] KIM JJ, TANNOCK IF. Repopulation of cancer cells during therapy: an important cause of treatment failure. Nat Rev Cancer 2005; 5: 516-525. http: //dx.doi.org/10.1038/nrc1650

[5] VIKTORSSON K, DE PETRIS L, LEWENSOHN R. The role of $\mathrm{p} 53$ in treatment responses of lung cancer. Biochem Biophys Res Commun 2005; 331: 868-880. http://dx.doi.org/10.1016/ j.bbrc.2005.03.192

[6] SU D, MA S, LIU P, JIANG Z, LV W, et al. Genetic polymorphisms and treatment response in advanced non-small cell lung cancer. Lung Cancer 2007; 56: 281-288. http: //dx.doi. org/10.1016/j.lungcan.2006.12.002

[7] SHIRAISHI K, KOHNO T, TANAI C, GOTO Y, KUCHIBA $A$, et al. Association of DNA repair gene polymorphisms with response to platinum-based doublet chemotherapy in patients with non-small-cell lung cancer. J Clin Oncol 2010; 28 : 4945-4952. http: //dx.doi.org/10.1200/JCO.2010.30.5334

[8] ZHOU C, REN S, ZHOU S, ZHANG L, SU C, et al. Predictive effects of ERCC1 and XRCC3 SNP on efficacy of platinumbased chemotherapy in advanced NSCLC patients. Jpn J Clin Oncol 2010; 40: 954-960. http: //dx.doi.org/10.1093/ijco/ hyq071

[9] LI F, SUN X, SUN N, QIN S, CHENG H, et al. Association between polymorphisms of ERCC1 and XPD and clinical response to platinum-based chemotherapy in advanced nonsmall cell lung cancer. Am J Clin Oncol 2010; 33: 489-494. http: //dx.doi.org/10.1097/COC.0b013e3181b9cedc

[10] SUN X, LI F, SUN N, SHUKUI Q, BAOAN C, et al. Polymorphisms in XRCC1 and XPG and response to platinum-based chemotherapy in advanced non-small cell lung cancer patients. Lung Cancer 2009; 65: 230-236. http: //dx.doi. org/10.1016/j.lungcan.2008.11.014

[11] LIN L, LIU X, SONG S, WANG S. [A study on the relationship between RRM1 single nucleotide polymorphisms and clinical characteristics in lung cancer patients.]. Zhongguo Fei Ai Za Zhi 2008; 11: 784-788.

[12] PAN JH, HAN JX, WU JM, SHENG LJ, HUANG HN. CYP450 polymorphisms predict clinic outcomes to vinorelbine-based chemotherapy in patients with non-small-cell lung cancer. Acta Oncol 2007; 46: 361-366. http: //dx.doi.org/10.1080/ $\underline{02841860600902197}$

[13] ALBEROLA V, SARRIES C, ROSELL R, TARON M, DE LAS PENAS R, et al. Effect of the methylenetetrahydrofolate reductase C677T polymorphism on patients with cisplatin/ gemcitabine-treated stage IV non-small-cell lung cancer. Clin 
Lung Cancer 2004; 5: 360-365. http: //dx.doi.org/10.3816/ CLC.2004.n.014

[14] FUJITA S, MASAGO K, HATACHI Y, FUKUHARA A, HATA A, et al. Genetic polymorphisms in the endothelial nitric oxide synthase gene correlate with overall survival in advanced nonsmall-cell lung cancer patients treated with platinum-based doublet chemotherapy. BMC Med Genet 2010; 11: 167. http: //dx.doi.org/10.1186/1471-2350-11-167

[15] MONCADA S, ERUSALIMSKY JD. Does nitric oxide modulate mitochondrial energy generation and apoptosis? Nat Rev Mol Cell Biol 2002; 3: 214-220. http: //dx.doi.org/10.1038/ $\underline{\operatorname{nrm} 762}$

[16] XU W, LIU LZ, LOIZIDOU M, AHMED M, CHARLES IG. The role of nitric oxide in cancer. Cell Res 2002; 12:311-320. http: //dx.doi.org/10.1038/sj.cr.7290133

[17] GELLER DA, NUSSLER AK, DI SILVIO M, LOWENSTEIN CJ, SHAPIRO RA, et al. Cytokines, endotoxin, and glucocorticoids regulate the expression of inducible nitric oxide synthase in hepatocytes. Proc Natl Acad Sci U S A 1993; 90: 522-526. http: //dx.doi.org/10.1073/pnas.90.2.522

[18] MOCELLIN S, BRONTE V, NITTI D. Nitric oxide, a double edged sword in cancer biology: searching for therapeutic opportunities. Med Res Rev 2007; 27: 317-352. http: //dx.doi. org/10.1002/med.20092

[19] WINK DA, VODOVOTZ Y, LAVAL J, LAVAL F, DEWHIRST $\mathrm{MW}$, et al. The multifaceted roles of nitric oxide in cancer. Carcinogenesis 1998; 19: 711-721. http://dx.doi.org/10.1093/ carcin/19.5.711

[20] SU X, TAKAHASHI A, GUO G, MORI E, OKAMOTO N, et al. Biphasic effects of nitric oxide radicals on radiation-induced lethality and chromosome aberrations in human lung cancer cells carrying different p53 gene status. Int J Radiat Oncol Biol Phys 2010; 77: 559-565. http://dx.doi.org/10.1016/ j.ijrobp.2009.12.059

[21] GRAY LH, GREEN FO, HAWES CA. Effect of nitric oxide on the radiosensitivity of tumour cells. Nature 1958; 182: 952-953. http: //dx.doi.org/10.1038/182952a0

[22] MITCHELL JB, WINK DA, DEGRAFF W, GAMSON J, KEEFER LK, et al. Hypoxic mammalian cell radiosensitization by nitric oxide. Cancer Res 1993; 53: 5845-5848.

[23] WARDMAN P, ROTHKAMM K, FOLKES LK, WOODCOCK M, JOHNSTON PJ. Radiosensitization by nitric oxide at low radiation doses. Radiat Res 2007; 167: 475-484. http: //dx.doi.org/10.1667/RR0827.1

[24] DE RIDDER M, VERELLEN D, VEROVSKI V, STORME G. Hypoxic tumor cell radiosensitization through nitric oxide. Nitric Oxide 2008; 19: 164-169. http: //dx.doi.org/10.1016/ j.niox.2008.04.015

[25] DE RIDDER M, VAN ESCH G, ENGELS B, VEROVSKI V, STORME G. Hypoxic tumor cell radiosensitization: role of the iNOS/NO pathway. Bull Cancer 2008; 95: 282-291.

[26] JANSSENS MY, VAN DEN BERGE DL, VEROVSKI VN, MONSAERT C, STORME GA. Activation of inducible nitric oxide synthase results in nitric oxide-mediated radiosensitization of hypoxic EMT-6 tumor cells. Cancer Res 1998; 58: 5646-5648.

[27] SINGH S, COWEN RL, CHINJE EC, STRATFORD IJ. The impact of intracellular generation of nitric oxide on the radia- tion response of human tumor cells. Radiat Res 2009; 171: 572-580. http: //dx.doi.org/10.1667/RR1640.1

[28] ADAMS C, MCCARTHY HO, COULTER JA, WORTHINGTON J, MURPHY C, et al. Nitric oxide synthase gene therapy enhances the toxicity of cisplatin in cancer cells. J Gene Med 2009; 11: 160-168. http: //dx.doi.org/10.1002/jgm.1280

[29] YASUDA H, YAMAYA M, NAKAYAMA K, SASAKI T, EBIHARA S, et al. Randomized phase II trial comparing nitroglycerin plus vinorelbine and cisplatin with vinorelbine and cisplatin alone in previously untreated stage IIIB/IV nonsmall-cell lung cancer. J Clin Oncol 2006; 24: 688-694. http: //dx.doi.org/10.1200/JCO.2005.04.0436

[30] MATTHEWS NE, ADAMS MA, MAXWELL LR, GOFTON TE, GRAHAM CH. Nitric oxide-mediated regulation of chemosensitivity in cancer cells. J Natl Cancer Inst 2001; 93: 1879-1885. http: //dx.doi.org/10.1093/jnci/93.24.1879

[31] SCHNEIDERHAN N, BUDDE A, ZHANG Y, BRUNE B. Nitric oxide induces phosphorylation of p53 and impairs nuclear export. Oncogene 2003; 22: 2857-2868. http: //dx.doi. org/10.1038/sj.onc.1206431

[32] WANG Z, COOK T, ALBER S, LIU K, KOVESDI I, et al. Adenoviral gene transfer of the human inducible nitric oxide synthase gene enhances the radiation response of human colorectal cancer associated with alterations in tumor vascularity. Cancer Res 2004; 64: 1386-1395. http: //dx.doi. org/10.1158/0008-5472.CAN-03-1307

[33] COOK T, WANG Z, ALBER S, LIU K, WATKINS SC, et al. Nitric oxide and ionizing radiation synergistically promote apoptosis and growth inhibition of cancer by activating p53. Cancer Res 2004; 64: 8015-8021. http: //dx.doi.org/10.1158/ 0008-5472.CAN-04-2212

[34] MATSUMOTO H, TAKAHASHI A, OHNISHI T. Nitric oxide radicals choreograph a radioadaptive response. Cancer Res 2007; 67: 8574-8579. http: //dx.doi.org/10.1158/0008-5472. CAN-07-1913

[35] FETZ V, BIER C, HABTEMICHAEL N, SCHUON R, SCHWEITZER A, et al. Inducible NO synthase confers chemoresistance in head and neck cancer by modulating survivin. Int J Cancer 2009; 124: 2033-2041. http: //dx.doi. org/10.1002/ijc. 24182

[36] YANG DI, YIN JH, MISHRA S, MISHRA R, HSU CY. NOmediated chemoresistance in C6 glioma cells. Ann N Y Acad Sci 2002; 962: 8-17.

[37] TOZER GM, EVERETT SA. Nitric oxide in tumour biology and cancer therapy. Part 1: Physiological aspects. Clin Oncol (R Coll Radiol) 1997; 9: 282-293. http: //dx.doi.org/10.1016/ S0936-6555(05)80061-5

[38] EISENHAUER EA, THERASSE P, BOGAERTS J, SCHWARTZ LH, SARGENT D, et al. New response evaluation criteria in solid tumours: revised RECIST guideline (version 1.1). Eur J Cancer 2009; 45: 228-247. http: //dx.doi.org/10.1016/ j.ejca.2008.10.026

[39] FENG WX, MOKROUSOV I, WANG BB, NELSON H, JIAO WW, et al. Tag SNP polymorphism of CCL2 and its role in clinical tuberculosis in Han Chinese pediatric population. PLoS One 2011; 6: e14652. http://dx.doi.org/10.1371/journal. pone. 0014652 
[40] BARRETT JC, FRY B, MALLER J, DALY MJ. Haploview: analysis and visualization of LD and haplotype maps. Bioinformatics 2005; 21:263-265. http: //dx.doi.org/10.1093/ bioinformatics/bth457

[41] CHOI JY, BARLOW WE, ALBAIN KS, HONG CC, BLANCO JG, et al. Nitric oxide synthase variants and disease-free survival among treated and untreated breast cancer patients in a Southwest Oncology Group clinical trial. Clin Cancer Res 2009; 15: 5258-5266. http: //dx.doi.org/10.1158/1078-0432. CCR-09-0685
[42] CHOI JY, LEE KM, NOH DY, AHN SH, LEE JE, et al. Genetic polymorphisms of eNOS, hormone receptor status, and survival of breast cancer. Breast Cancer Res Treat 2006; 100: 213-218. http: //dx.doi.org/10.1007/s10549-006$\underline{\text { 9245-5 }}$

[43] RIENER EK, HEFLER LA, GRIMM C, GALID A, ZEILLINGER R, et al. Polymorphisms of the endothelial nitric oxide synthase gene in women with vulvar cancer. Gynecol Oncol 2004; 93: 686-690. http: //dx.doi. org/10.1016/j.ygyno.2004.03.030 